Data from different sources have different applications: for a large healthcare system or insurance organization looking to allocate resources and determine policy for a population, we commend the use of the Kaiser data. For a surgical practitioner counseling his or her patients, we commend data such as our own.

We share with our crosstown colleagues the clinical conclusion that expectant management of HSIL is reasonable, based on evidence and experience. We wonder if we can now call it San Diego rather than expectant management, to use a sunnier and less pregnant term! We also share with everyone in the field, including advocates of HSIL ablation with whom we disagree, the conviction that people at high risk for anal cancer should be followed regularly by practitioners accustomed to examining the anus. Indurated or ulcerated lesions should be biopsied so that early stage cancers can be detected and cured.

Bard C. Cosman, M.D., M.P.H. VA San Diego Healthcare System, San Diego, California

Sonia L. Ramamoorthy, M.D.

Luis C. Cajas-Monson, M.D. Division of Colon and Rectal Surgery Department of Surgery University of California San Diego School of Medicine La Jolla, California

\section{The Authors Reply}

To the Editor-In his letter, Dr Tomassi describes his study from Kaiser Permanente Southern California, in which the investigators "expectantly managed" 378 patients with high-grade anal dysplasia, demonstrating an incidence rate of $0.19 \%$ per patient-year for anal squamous cell carcinoma (SCC). This rate is lower than the rate we identified using the population-based cancer registries of the Surveillance, Epidemiology, and End Results (SEER) Program of the National Cancer Institute. It must be emphasized that Dr Tomassi's data derive from Kaiser Permanente billing codes, and therefore all included patients are medically insured. As Dr Tomassi states in his letter, surveillance of patients at high risk for anal SCC is provided by "general and colorectal surgeons, infectious disease specialists, family medicine and internal medicine physicians, and gynecologists." The Kaiser Permanente data come from a large integrated managed care group that receives high marks for quality of care with an emphasis on preventive care and access to care. This likely represents a higher level of longitudinal care than most patients in the United States are fortunate enough to receive. Our SEER data are derived from $34.6 \%$ of the US population, irrespective of insurance status, and are

Dis Colon Rectum 2019; 62: e36

DOI: 10.1097/DCR.0000000000001392

(C) The ASCRS 2019 the most representative sample of the overall state of cancer care in this country. It also must be noted that SEER includes patients with any neoplasia, not just invasive cancers.

Importantly, although Dr Tomassi states that the Kaiser cohort is a "pure expectant management cohort," he notes that "patients with grossly abnormal or symptomatic lesions" were treated with "punch biopsy, excisional biopsy, or fulguration." It is unclear exactly what other treatments were rendered based on the data presented. In addition, Dr Tomassi describes general surveillance procedures for high-risk patients ("digital rectal examination and anoscopy at interval times ranging from 3 to 12 months"), but the actual frequency and type of surveillance each patient received was not recorded. Ultimately and most importantly, the accuracy of the codes for anal intraepithelial neoplasia (AIN) is also unclear. Although the investigators verified the abstracted data with Kaiser Permanente Southern California registries for HIV, anal SCC, and cervical cancer, there does not appear to be an equivalent registry for the validation of anal dysplasia diagnoses. In contrast, SEER is a curated cancer registry not based on billing data but rather standard pathology report abstraction and rigorous monitoring processes to ensure high-quality data.

We are pleased that our article has started such a rich discussion of the appropriate management and surveillance of patients with high-grade anal dysplasia. With this new wealth of data, it is imperative to consider the advantages and disadvantages of each data source, from single institutions to population-level databases, and billing data to cancer registries. Ultimately, although Dr Tomassi found a lower rate of subsequent anal SCC in patients with high-grade anal dysplasia than our study, we all agree that the rate is significant enough to warrant surveillance of patients with AIN III. None of our studies can identify the single best algorithm for surveillance, yet all our studies report concerning trends for patients with AIN.

Grace C. Lee, M.D. Rocco Ricciardi, M.D. Massachusetts General Hospital Boston, Massachusetts

\section{New Attempt to Reach a Common Sense in Pilonidal Sinus Therapy}

To the Editor-The new Clinical Practice Guidelines of the American Society of Colon and Rectal Surgeons have now been published, ${ }^{1}$ and the team headed by Scott Steele can be congratulated on their extensive and meticulous work,

Dis Colon Rectum 2019; 62: e36-e37

DOI: 10.1097/DCR.0000000000001394

(C) The ASCRS 2019 


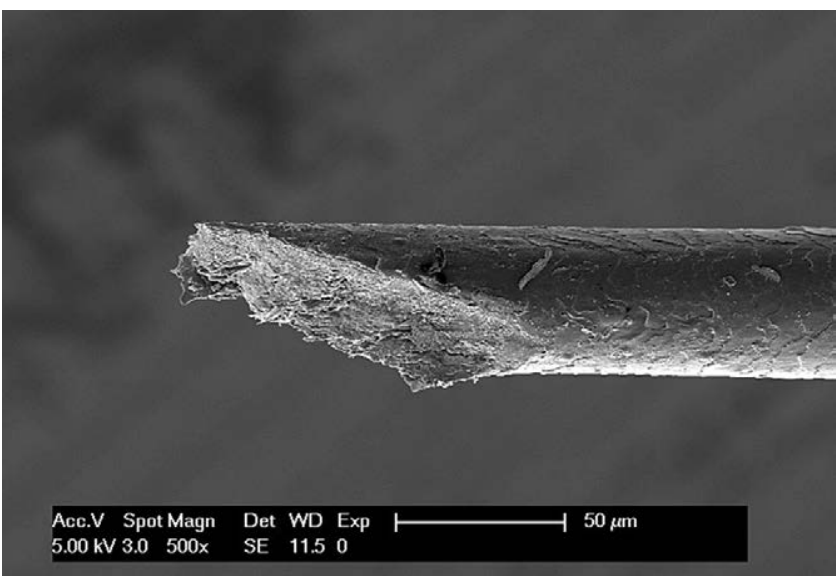

FIGURE 1. Scanning electron microscopy picture of a razor-sharp cut hair end (courtesy of Norbert Pütz, University of the Saarland, Homburg/Saar).

which addresses most of the questions associated with pilonidal sinus surgery, improving the original Society guidelines grossly. ${ }^{2}$ They acknowledge that stronger, razor-sharp cut hairs from the head (as seen in Figure 1), accumulating between the buttocks and piercing the healthy skin, ${ }^{3}$ may be the source of pilonidal sinus disease. ${ }^{4,5} \mathrm{We}$ agree with the majority of conclusions in the guidelines, but several topics are controversial at best.

\section{Depilation}

The authors correctly point out that razor depilation increases the long-term recurrence rate. ${ }^{6}$ So why do they recommend it, as it doubles the recurrence rate?

Occipital hair is found in the sinus nest; so why is intergluteal depilation important nevertheless? Intergluteal hair is a powerful catching zone, where cut hair from the head is caught, retained, and waiting to be drilled into the healthy skin by regular muscular movements. Thus, depilation of the cranial intergluteal fold may reduce recurrence rate, because it decreases contact time. Depilation creams may harm the skin with alkaline ingredients, thus harming the natural barrier. Here, laser is of interest. However, even a perfect laser depilation will not remove the cut hair per se, so no depilation treatment alone will ever achieve a zero recurrence rate. This could have been emphasized by the authors.

\section{Hygiene}

We are not at all happy that the new guidelines still address hygiene. ${ }^{1}$ Speaking bluntly, this is an insult to our patients, who already suffer enough from their disease. The majority of young adolescents who we treat are seen shortly after puberty, when they discover sexuality. Many of them take showers 1 to 2 times a day. It has been shown that neither long-standing pus, nor feces, nor urine promotes or exaggerates pilonidal disease. ${ }^{7}$ Regular bathing or showering may lower pilonidal recurrence, however, ${ }^{8,9}$ because it reduces the number of cut hair fragments (Fig. 1) in the intergluteal cleft. So, yes, showering and bathing are useful to remove cut hair and clean open wounds if present, but hygienic enforcement has no place in a national clinical practice recommendation, because it punishes a majority of patients for something that is not their fault.

\section{Corrosive Substances}

The idea of corrosive tract and nest obliteration is an appealing one, and the injection of radioactive substances such as Thorium X, Lipoidol, and fuming nitric acid has been tried in the past. It has been discarded though. Phenol is commonly used in the Indo-Arab region but is forbidden in Germany because of its corrosive and potentially embryotoxic effects. Long-term recurrence rates are dismal, ${ }^{10}$ with a 5-year recurrence rate for phenol in excess of $40 \% .{ }^{10}$ Differences in application (crystalline, gel, or fluid) and concentration $(25 \%-80 \%)^{11}$ may have an influence on efficacy but are not addressed in the new guidelines.

\section{Recurrence Rates}

Unfortunately the authors of the guidelines cite Stansby for his extended follow-up and give recurrence rates of $0 \%$ to $11 \% .{ }^{1}$ Stansby's recurrence rate is $10(15 \%)$ of 65 in primary open treatment and 12 (12\%) of 104 in phenol treatment after a mean 8 months of follow-up. ${ }^{12}$ Thus his (less than) 1 -year recurrence rate is $12 \%$, which is impressively higher than any other pilonidal sinus disease therapy's 1 -year recurrence rate. ${ }^{10}$ In our opinion, durable healing is not present at all, and the strong recommendation based on moderate evidence should be reconsidered.

In conclusion, it is a daunting task to elaborate a clinical practice guideline and to come to a sound conclusion. We thank Johnson et $\mathrm{al}^{1}$ for their substantial work and efforts. Taking the above-mentioned remarks into account, their recommendations will be well received.

\section{REFERENCES}

1. Johnson EK, Vogel JD, Cowan ML, Feingold DL, Steele SR; Clinical Practice Guidelines Committee of the American Society of Colon and Rectal Surgeons. The American Society of Colon and Rectal Surgeons' clinical practice guidelines for the management of pilonidal disease. Dis Colon Rectum. 2019;62:146-157.

2. Steele SR, Perry WB, Mills S, Buie WD; Standards Practice Task Force of the American Society of Colon and Rectal Surgeons. Practice parameters for the management of pilonidal disease. Dis Colon Rectum. 2013;56:1021-1027.

3. Bosche F, Luedi MM, van der Zypen D, Moersdorf P, Krapohl B, Doll D. The hair in the sinus: sharp-ended rootless head hair fragments can be found in large amounts in pilonidal sinus nests. World J Surg. 2018;42:567-573.

4. Doll D, Bosche F, Hauser A, et al. The presence of occipital hair in the pilonidal sinus cavity-a triple approach to proof. Int $J$ Colorectal Dis. 2018;33:567-576. 
5. Doll D, Bosche FD, Stauffer VK, et al. Strength of occipital hair as an explanation for pilonidal sinus disease caused by intruding hair. Dis Colon Rectum. 2017;60:979-986.

6. Petersen S, Wietelmann K, Evers T, Hüser N, Matevossian E, Doll D. Long-term effects of postoperative razor epilation in pilonidal sinus disease. Dis Colon Rectum. 2009;52:131-134.

7. Doll D, Luedi MM, Wieferich K, van der Zypen D, Maak M, Glanemann M. Stop insulting the patient: neither incidence nor recurrence in pilonidal sinus disease is linked to personal hygiene. Pilonidal Sinus Journal. 2015;1:11-19.

8. Harlak A, Mentes O, Kilic S, Coskun K, Duman K, Yilmaz F. Sacrococcygeal pilonidal disease: analysis of previously proposed risk factors. Clinics (Sao Paulo). 2010;65:125-131.

9. Bolandparvaz S, Moghadam Dizaj P, Salahi R, et al. Evaluation of the risk factors of pilonidal sinus: a single center experience. Turk J Gastroenterol. 2012;23:535-537.

10. Stauffer VK, Luedi MM, Kauf P, et al. Common surgical procedures in pilonidal sinus disease: a meta-analysis, merged data analysis, and comprehensive study on recurrence. Sci Rep. 2018;8:3058.

11. Emiroğlu M, Karaali C, Esin H, Akpınar G, Aydın C. Treatment of pilonidal disease by phenol application. Turk J Surg. 2017;33:5-9.

12. Stansby G, Greatorex R. Phenol treatment of pilonidal sinuses of the natal cleft. Br J Surg. 1989;76:729-730.

Dietrich Doll, M.D., Ph.D. Directorate of Trauma and Burns, Chris Hani Baragwanath Academic Hospital, University of Witwatersrand Medical School, Johannesburg, Republic of South Africa Department of Surgery, Catholic Clinics Oldenburger Münsterland gGmbH, Saint Mary's Hospital Vechta, Teaching Hospital of the Medizinische Hochschule Hannover University, Vechta, Germany Markus M. Luedi, M.D., M.B.A. Department of Anaesthesiology and Pain Medicine, Inselspital, Bern University Hospital, Bern, Switzerland

\section{The Authors Reply}

To the Editor-We appreciate the letter to the editor from Drs Doll and Luedi regarding the most recent update to the American Society of Colon and Rectal Surgeons' Clinical Practice Guidelines for the Management of Pilonidal Disease. ${ }^{1}$ The authors raise the issue of attempting to reach a "common sense" approach in the management of pilonidal sinus therapy. The authors have certain interest and expertise in the area; in fact, much of the information and articles they cite (many of their own work) were reviewed and/or cited by the Clinical Practice Guidelines committee for this update.

Specifically, the authors note the controversies in the management of pilonidal disease, somewhat interesting

Dis Colon Rectum 2019; 62: e38

DOI: 10.1097/DCR.0000000000001393

(C) The ASCRS 2019 considering its relative common incidence. Although the authors note concern regarding depilation and hygiene, as well as intriguing more novel therapies such as corrosive substances, we are subtly reminded about the paucity of high-quality literature on this topic. One of the difficulties in preparing these and most surgical guidelines is the inherent limitations in the primary source literature, along with the varying experience and outcomes of individual authors within their own personal series.

We are again reminded that the American Society of Colon and Rectal Surgeons' Clinical Practice Guidelines (CPG) committee is charged with the task of identifying areas of colorectal surgery that require guidance for evidence-based practice. The committee collects pertinent literature, reviews and grades it according to guidelines set out by the GRADE group, and applies it to specific standards of practice. We strive to assess the available literature on the topic of interest fairly, and present it in an unbiased fashion. The reference list is not meant to be all inclusive, but rather to be broad-based. Our goal is to provide guidance to help the clinician formulate a treatment plan that is effective and safe for the individual patient. This will always require judgment on the part of the clinician.

We agree that recurrence rates in patients with pilonidal disease are a function of time, and that extended follow-up is required. We also agree that there is a spectrum of response to both nonoperative and operative interventions. In light the available literature and widespread results, the determination of causation versus association is difficult to delineate definitively. If the parameter is read carefully, there is no mention of poor hygiene as a cause of pilonidal disease. However, any and all skin wounds including pilonidal should be subject to cleaning and proper hygiene to enhance healing. Regarding more novel therapies, we await further experience with longer follow-up to make definitive recommendations.

We would like to thank Drs Doll and Luedi for their thoughtful commentary. As with all CPGs, as additional evidence comes to light, future CPGs will review the relevant literature and adjust any recommendations as indicated.

\section{REFERENCES}

1. Johnson EK, Vogel JD, Cowan ML, Feingold DL, Steele SR; Clinical Practice Guidelines Committee of the American Society of Colon and Rectal Surgeons. The American Society of Colon and Rectal Surgeons' Clinical Practice Guidelines for the Management of Pilonidal Disease. Dis Colon Rectum. 2019;62:146-157.

Eric K. Johnson, M.D.

Scott R. Steele, M.D., M.B.A.

On behalf of the Clinical Practice Guidelines Committee of the American Society of Colon and Rectal Surgeons 